Dementia

and Geriatric

Cognitive Disorders
Dement Geriatr Cogn Disord 2017;43:247-258

\title{
Lower Visual Avoidance in Dementia Patients Is Associated with Greater Psychological Distress in Caregivers
}

\author{
Marcela C. Otero Robert W. Levenson \\ Institute of Personality and Social Research, University of California, Berkeley, CA, USA
}

\section{Keywords}

Emotion regulation - Caregiver psychological health · Frontotemporal dementia .

Alzheimer disease

\begin{abstract}
Caring for a spouse with dementia can lead to increased health problems in caregivers. The present study examined whether patient deficits in visual avoidance, a common form of emotion regulation, are related to greater psychological distress in caregivers. Participants were $43 \mathrm{Alz}$ heimer disease (AD) patients, 43 behavioral variant frontotemporal dementia (bvFTD) patients, and their spousal caregivers. Patient visual avoidance (e.g., gaze aversion) was measured using behavioral coding of head, body, and eye position while viewing a disgusting film. Caregiver psychological distress was measured using a standard self-report symptom inventory. Lower use of visual avoidance by patients was associated with greater psychological distress in their caregivers. This relationship was partially mediated by patient overall emotional functioning (as reported by caregivers), such that patients with less visual avoidance were seen as having worse emotional functioning, which in turn related to greater caregiver psychological distress. Dementia diagnosis moderated this effect, with diminished patient visual avoidance particularly detrimental to psychological distress of bvFTD caregivers. Findings suggest that the use of visual avoidance may serve as a marker of overall emotional functioning in patients and that preservation of this emotion regulatory behavior may help reduce the negative effects of caregiving.
\end{abstract}

(c) 2017 S. Karger AG, Basel

\section{Introduction}

Neurodegenerative diseases are expected to affect over 115 million individuals world wide by 2050 [1]. Among these diseases, Alzheimer disease (AD) accounts for $60-70 \%$ of all dementias and primarily produces memory loss and declines in visual-spatial processing 
[2-4]. Frontotemporal dementia is less common overall, but its prevalence is similar to AD among early onset dementias [5-7]. Behavioral variant frontotemporal dementia (bvFTD) is characterized by progressive behavioral changes including changes in personality, emotional blunting, increased disinhibition, and inappropriate interpersonal behavior [8, 9]. Given the progressive and debilitating nature of these diseases, patients typically come to depend on caregivers to provide for their psychological and physical needs.

Caring for a loved one with dementia is an important and meaningful part of family life. However, it is associated with increased burden that can lead to a host of negative outcomes including increases in physical illness, mental illness (especially depression and anxiety), and mortality [10-13]. Notably, not all caregivers suffer these negative consequences. Thus, it is important to identify factors in the patient, the caregiver, and in the patient-caregiver relationship that create greater vulnerability or resilience to the negative effects of caregiving. Previous research has indicated that declines in patients' emotional functioning are strongly linked with increased psychological distress in caregivers [14-16].

\section{Visual Avoidance: An Important Form of Emotion Regulation}

Emotion regulation can take a number of different forms [17]. Most research on emotion regulation has focused on behavioral suppression (limiting expressive signs of emotion) and reappraisal (thinking about potential emotion-elicitors in different ways) [18]. One of the most common and powerful forms of emotion regulation is visual avoidance. Limiting visual processing of an unpleasant emotion-eliciting stimulus (e.g., by looking away, closing one's eyes) reduces visual sensory input and thus decreases the capacity of the stimulus to initiate a full-blown emotional response. Previous work from our laboratory has shown that dementia patients show deficits in their use of visual avoidance (Otero and Levenson, under review). Given that the ability to regulate emotion is a cornerstone of successful emotional functioning, diminished visual avoidance in patients may be linked to negative consequences for caregiver psychological distress by virtue of related deficits in both emotion regulation and in overall emotional functioning.

\section{Present Study}

The present study sought to test the hypothesis that less use of visual avoidance by patients would predict greater psychological distress in caregivers. Assuming this association was found, we planned to determine whether it was mediated by caregivers' ratings of patients' emotion functioning (reflecting our view that loss of visual avoidance in dementia patients is a marker of declining overall emotional functioning). Since cognitive and neuropsychiatric deficits are so prominent in $\mathrm{AD}$ and other dementias, we also planned to test an alternative model that linked patients' cognitive functioning and neuropsychiatric symptomatology with caregiver psychological distress. Because losses of emotional functioning are so prominent in bvFTD $[19,20]$, we hypothesized that the association between deficits in visual avoidance and caregiver psychological distress would be greater in bvFTD caregivers than AD caregivers.

\section{Method}

Participants

A total of 86 patients with dementia (43 with AD and 43 with bvFTD) and their caregivers were recruited through the Memory and Aging Center (MAC) at the University of California, San Francisco, CA, USA. AD and bvFTD diagnoses were based on a comprehensive assessment that included a clinical interview, neurological and neuropsychological testing, and structural MRIs. AD patients met the Neurological and Communicative Disorders and Stroke and Alzheimer's Disease and Related Disorders Association criteria [4], and bvFTD 


\section{Dementia}

and Geriatric Cognitive Disorders

Table 1. Interrater reliabilities for individual visual avoidance codes

\begin{tabular}{l|l}
\hline \multicolumn{2}{l}{ Dement Geriatr Cogn Disord 2017;43:247-258 } \\
\hline DOI: 10.1159/000468146 & $\begin{array}{l}\text { C 2017 S. Karger AG, Basel } \\
\text { www.karger.com/dem }\end{array}$ \\
\hline
\end{tabular}

Otero and Levenson: Lower Visual Avoidance in Dementia Patients Is Associated with Greater Psychological Distress in Caregivers

\begin{tabular}{ll}
\hline Behavior & Interrater ICC \\
\hline Blinks & 1.00 \\
Head turn & 0.76 \\
Head down & 0.85 \\
Head up & 0.58 \\
Squints & 0.99 \\
Eyes closed & 0.91 \\
Eyes covered & 1.00 \\
Gaze aversion & 0.73 \\
Head shake & 0.94 \\
\hline
\end{tabular}

patients met the Neary criteria [21]. Caregivers were all either spouses or domestic partners who served as the patient's primary caregiver.

\section{Procedure}

Patient Laboratory Assessment

Patients and their caregivers came to the Berkeley Psychophysiology Laboratory at the University of California, Berkeley, CA, USA, for a daylong session designed to provide a comprehensive assessment of emotional functioning [22]. The current study focused on a task in which patients: (a) sit quietly for a 60-second baseline period; (b) watch a 101-second film clip taken from the television show, Fear Factor, which shows a man sucking digestive fluids from cow intestines and has been found to elicit disgust expressive behaviors and visual avoidance in neurologically healthy adults [23, Otero and Levenson, under review]; (c) sit quietly for a 60-second post-film period; and (d) answer a series of questions assessing their comprehension and memory of the film's content and their emotional experience while viewing the film. Throughout the task, participants were videotaped for later coding of visual avoidance behaviors and a number of psychophysiological measures were obtained (these physiological data were not used in the present study). At the end of the day, patients gave written consent for the use of their video recordings and were paid USD 30 for their participation.

\section{Caregiver Assessment}

While patients participated in the laboratory assessment, caregivers completed self-report questionnaires that assessed their psychological distress and various areas of patient functioning.

\section{Measures}

Visual Avoidance

Patient behavior was coded using a behavioral coding system developed for this study. The coding system consisted of 9 head, body, and eye movements identified in the literature as indicating attentional disengagement (i.e., visual avoidance) [24-26]. The 9 visual avoidance codes were as follows: (a) head turning, (b) head down, (c) head up, (d) headshakes, (e) gaze aversion, (f) blinks, (g) eyes closed, (h) eyes covered, and (i) squint. Four of the 9 codes (i.e., head turn, head down, head up, and head shakes) were rated using a 4-point intensity scale with $0=$ no code, $1=$ slight intensity, $2=$ moderate intensity, and $3=$ extreme intensity. Two codes (i.e., eyes closed and eyes covered) were rated using a 3-point intensity scale with $0=$ no code, 1 = partial eye coverage, and 2 = complete eye coverage. Three codes (gaze aversion, blinks, and squints) were measured by tallying the total number of times the code occurred. Participants' second-bysecond behavior was coded throughout the trial.

Coders were 2 male and 1 female upper level undergraduate research assistants who were naïve to the study's goal and patient diagnoses. Each coder underwent 6 weeks of training consisting of formal instruction based on a coding manual, weekly practice coding assignments, bi-weekly 1-hour meetings to discuss coding disagreement, and a final coding examination. Interrater reliability at the end of training was high (intraclass correlation coefficient $=0.90$ ). To assess reliability, all 3 coders coded $20 \%$ of the study sample. Interrater reliability for the study was good (intraclass correlation coefficient $=0.86$ ). Interrater reliability for individual codes is shown in Table 1 . 
Dementia

Cognitive Disorders

\begin{tabular}{l|l}
\hline Dement Geriatr Cogn Disord 2017;43:247-258 \\
\hline DOI: 10.1159/000468146 & $\begin{array}{l}\text { (c) 2017 S. Karger AG, Basel } \\
\text { www.karger.com/dem }\end{array}$ \\
\hline
\end{tabular}

Otero and Levenson: Lower Visual Avoidance in Dementia Patients Is Associated with Greater Psychological Distress in Caregivers

Caregiver Psychological Distress

Caregiver psychological distress was assessed using the Symptom Checklist 90-Revised (SCL-90-R) [27]. The SCL-90-R is a 90-item questionnaire that assesses 9 domains of psychopathology (somatization, obsessive-compulsive, interpersonal sensitivity, depression, anxiety, hostility, phobic anxiety, paranoid ideation, and psychoticism). For each item, caregivers rated themselves on a 5-point scale of distress from 0 (none) to 4 (extreme). For the present study, our primary measure was the Global Severity Index (GSI), which is the average score of all 90 items and is thought to be a good indicator of psychological distress [28].

Patient Dementia Severity

Dementia severity was assessed using the Clinical Dementia Rating (CDR) Scale [29]. Caregivers rated patients' level of impairment in several domains found to be impaired in dementia (memory, problemsolving, orientation, judgment, community affairs, home and hobbies, as well as personal care). A CDR Box Score was created for each participant by summing the total scores for each domain, with higher scores indicating greater dementia severity.

Patient Emotional Functioning

Patient emotional functioning was assessed using the Caregiver Assessment of Socioemotional Functioning (CASEF), a 44-item questionnaire we developed to assess various domains of emotional and social functioning [30]. For each item, caregivers rated the patient's tendency to engage in certain behaviors during the past month on a 5-point scale from 0 (not at all) to 4 (a lot). For the present study, we utilized the 33-item emotional functioning subscale (e.g., "Patient expresses anger"; "Patient is warm/affectionate toward spouse/partner/family"). Interitem reliability for this subscale was high (Cronbach's $\alpha=0.94$ ).

\section{Patient Cognitive Functioning}

Patient cognitive functioning was assessed using the cognitive subscales of the CDR (i.e., memory, orientation, and problem-solving). Caregivers rated patients' cognitive functioning on a 0-3 scale, with higher scores representing greater levels of cognitive impairment. A composite cognitive functioning score was computed by averaging the total score of each cognitive domain.

Patient Neuropsychiatric Symptomatology

Patient neuropsychiatric symptoms were assessed using a clinician rated Neuropsychiatric Inventory (NPI) total score [31]. The NPI total score consists of the sum of 12 subscale scores including delusions, hallucinations, dysphoria, irritability, agitation, anxiety, apathy, disinhibition, euphoria, aberrant motor behavior, nighttime behavior disturbances, and appetite and eating abnormalities.

\section{Caregiver Well-Being}

Caregiver well-being was assessed using the Medical Outcomes Study 36-Item Short Form Health Survey (SF-36) [32]. The SF-36 consists of 8 subscales including: (1) physical functioning; (2) role limitation due to physical health problems; (3) role limitations due to emotional problems; (4) bodily pain; (5) social functioning; (6) general mental health (psychological well-being and distress); (7) vitality (energy and fatigue); and (8) general health perceptions. A SF-36 composite score was computed by averaging the scores of all 8 subscales.

\section{Data Reduction}

Visual Avoidance

Preliminary analyses revealed that 2 of the 9 codes (squints and eyes covered) rarely occurred (squint in $4.2 \%$ of participants and eyes covered in $0.8 \%$ of participants). Thus, they were excluded from further analyses. This left 7 visual avoidance codes: head turning, head up, head down, gaze aversion, blink, eye closed, and headshake.

An overall visual avoidance score for each patient was computed as follows. First, for each of the 7 coded behaviors, the intensity scores for each second of the film were summed and divided by 101 (the duration of the film in seconds) producing an average intensity per second. These average intensity scores were then converted to $z$ scores (using the mean and standard deviation from the entire sample) and summed to create a single composite score of visual avoidance for each participant. The reliability of this overall score was moderate (Cohen's $\alpha=0.53$ ) [33]. To control for type I error, we conducted our primary analyses using this overall score, but also conducted follow-up analyses using the individual codes. 
Dementia

Cognitive Disorders

Table 2. Demographic and clinical variables

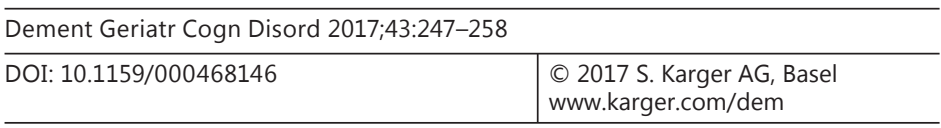

Otero and Levenson: Lower Visual Avoidance in Dementia Patients Is Associated with Greater Psychological Distress in Caregivers

\begin{tabular}{lcc}
\hline & AD & bvFTD \\
\hline Number of patients & 43 & 43 \\
Male/female sex, $n$ & $18 / 25$ & $15 / 28$ \\
Mean age, years (SD) & $61.81(8.22)$ & $61.07(8.24)$ \\
Mean CDR-BS (SD) & $4.68(2.17)$ & $6.58(3.04)$ \\
Mean SF-36 (SD) & $76.72(13.87)$ & $72.94(14.58)$ \\
Mean NPI total score, (SD) & $18.33(15.44)$ & $46.19(19.25)$ \\
\hline
\end{tabular}

CDR-BS, Clinical Dementia Rating Scale Box Score; SF-36, Medical Outcomes Study 36-Item Short Form Health Survey; NPI, Neuropsychiatric Inventory.
Table 3. Patient visual avoidance composite (entire task), patient overall emotional and cognitive functioning

\begin{tabular}{lrr}
\hline & AD & \multicolumn{1}{l}{ bvFTD } \\
\hline Visual avoidance & $1.16(4.42)$ & $-1.16(1.85)$ \\
CASEF emotional functioning subscale & $2.06(0.61)$ & $1.25(0.60)$ \\
CDR cognitive subscales average & $82(0.34)$ & $1.07(0.42)$ \\
\hline
\end{tabular}

CASEF, Caregiver Assessment of Socioemotional Functioning; CDR, Clinical Dementia Rating.

\section{Results}

\section{Demographic and Clinical Variables}

A $\chi^{2}$ test was used to examine the distribution of males and females within the diagnostic groups. No sex differences were found, $\chi^{2}(2, n=86)=0.44$, ns. An analysis of variance (ANOVA) was used to examine age differences between the groups. No age differences were found between AD and bvFTD patients, $F(1,84)=11.91$, ns. ANOVAs were also used to examine diagnostic group differences in patient disease severity and neuropsychiatric symptomatology, as well as overall caregiver health. BvFTD patients showed significantly greater disease severity, $F(1,84)=77.71, p=0.001$, and NPI total scores, $F(1,82)=53.52, p<0.001$, compared to AD patients. No differences were found between bvFTD and AD caregiver health, $F(1,80)=1.45$, ns (see Table 2 for means and standard deviations).

\section{Patient Visual Avoidance and Caregiver Psychological Distress}

\section{Caregiver Psychological Distress}

We conducted a multiple linear regression analysis to examine whether visual avoidance in patients predicted caregiver psychological distress. To control for dementia severity, we entered the CDR Box Score in the first step. In the second step, we entered patient visual avoidance, which accounted for significant additional variance $(F$ change $(1,83)=5.80, p=$ $0.018)$. In this second step, dementia severity did not predict caregiver psychological distress $(t(83)=0.23, p=0.816)$, but less patient visual avoidance significantly predicted greater caregiver psychological distress $(\beta=-0.26, t(83)=-2.41, p=0.018)$ (see Table 3 for patient visual avoidance means and standard deviations).

\section{Indirect Effect of Patient Emotional Functioning}

Having established an association between lower patient visual avoidance and greater caregiver psychological distress, we conducted a mediation analysis to test whether this asso- 
Otero and Levenson: Lower Visual Avoidance in Dementia Patients Is Associated with Greater Psychological Distress in Caregivers

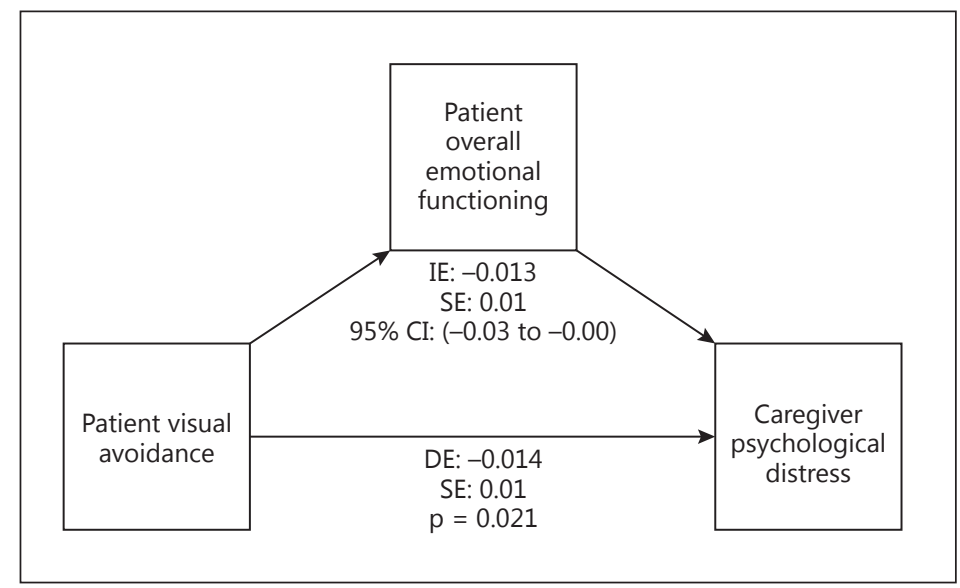

Fig. 1. The relationship between patient visual avoidance and caregiver psychological distress was indirectly transmitted by patient overall emotional functioning, even when controlling for caregiver well-being. There was also a direct effect of patient visual avoidance on caregiver psychological distress, independent of patient emotional functioning. Data represent indirect and direct effects and standard errors using 10,000 bootstrap samples to obtain bias-corrected and accelerated $95 \%$ confidence intervals and p values.

ciation was mediated via caregiver reports of lower patient emotional functioning. This analysis utilized a least squares path analysis in which patient visual avoidance was the independent variable, caregiver psychological distress was the outcome variable, caregiver ratings of patient emotional functioning was the mediator, and caregiver well-being (SF-36 total score) was a covariate.

Results revealed a significant indirect effect of patient emotional functioning on patient visual avoidance and caregiver psychological distress, [IE] $=-0.01, \mathrm{SE}=0.01,95 \% \mathrm{CI}-0.03$ to -0.00 , indicating that patients who showed less visual avoidance in the laboratory assessment were seen by their caregivers as having lower levels of overall emotional functioning which in turn was associated with greater caregiver psychological distress, even when controlling for overall caregiver well-being (Fig. 1). There was also evidence for a direct effect of less patient visual avoidance on greater caregiver psychological distress, $[\mathrm{DE}]=-0.01, \mathrm{SE}=0.01$, $95 \% \mathrm{CI}-0.03$ to $0.00, p=0.021$, indicating that some of the association between lower visual avoidance by patients and greater caregiver psychological distress may be transmitted in other ways besides lower patient emotional functioning.

Other possible candidates accounting for the association between lower patient visual avoidance and greater caregiver psychological distress are lower patient cognitive functioning and greater patient neuropsychiatric symptomatology. To test this possibility, we conducted a multiple mediation analysis in which patient visual avoidance was the independent variable, caregiver psychological distress was the dependent variable, and all 3 patient factors (emotional functioning, cognitive functioning, and neuropsychiatric symptomatology) were mediators. This analysis revealed that the indirect effect of lower patient emotional functioning remained significant even when patient cognitive functioning and neuropsychiatric symptomatology were entered into the model, [IE] $=-0.01, \mathrm{SE}=0.01,95 \%$ $\mathrm{CI}-0.03$ to -0.00 . A comparison of indirect effects showed that the indirect effect of lower patient emotional functioning was significantly stronger than both the indirect effect of lower patient cognitive functioning, [contrast IE] $=-0.01, \mathrm{SE}=0.01,95 \% \mathrm{CI}-0.03$ to -0.00 , and greater neuropsychiatric symptoms, [contrast IE] $=-0.01, \mathrm{SE}=0.01,95 \% \mathrm{CI}-0.03$ to -0.00 . Thus, we conclude that in this sample of patients, lower patient emotional functioning is more 
Dementia

and Geriatric

Table 4. Caregiver psychological distress, anxiety, and depression

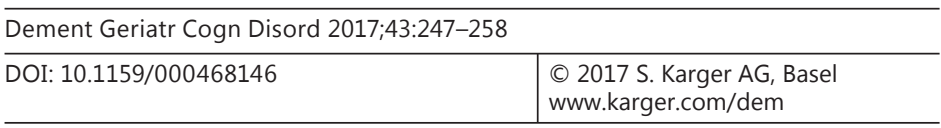

Otero and Levenson: Lower Visual Avoidance in Dementia Patients Is Associated with Greater Psychological Distress in Caregivers

\begin{tabular}{lll}
\hline & AD & bvFTD \\
\hline Mean SCL-90-R-GSI (SD) & $0.56(0.23)$ & $0.71(0.25)$ \\
Mean SCL-90-R DEP (SD) & $0.71(0.36)$ & $0.95(0.38)$ \\
Mean SCL-90-R-ANX (SD) & $0.45(0.35)$ & $0.65(0.35)$ \\
\hline
\end{tabular}

SCL-90-R-GSI, Symptom Checklist-90-Revised, Global Severity Index; SCL-90-R-DEP, Symptom Checklist-90-Revised, Depression Subscale; SCL-90-R-ANX, Symptom Checklist-90-Revised, Anxiety Subscale.

important than lower patient cognitive functioning and greater neuropsychiatric symptomatology for understanding the relationship between lower patient visual avoidance and greater caregiver psychological distress.

\section{Moderation by Diagnosis}

To determine whether patient diagnosis moderated the effect of patient visual avoidance on caregiver psychological distress, we included the interaction of diagnosis and patient visual avoidance behavior in a regression analysis (patient visual avoidance was mean centered and patient diagnosis was coded as $\mathrm{AD}=0$, bvFTD $=1$ ). Results revealed a significant interaction between patient diagnosis and visual avoidance behaviors $(\beta=-0.05, t(82)=$ $-2.39,95 \% \mathrm{CI}-0.09$ to $-0.01, p=0.020$ ), such that lower patient visual avoidance was associated with greater caregiver psychological distress in bvFTD couples $(t(82)=-2.84,95 \% \mathrm{CI}$ -0.09 to $-0.02, p=0.006)$, but not in AD couples, $(t(82)=-0.59,95 \% \mathrm{CI}-0.02-0.1, p=0.559)$. These results indicate that the associations among lower visual avoidance in patients and greater psychological distress in caregivers are particularly pronounced for bvFTD patients and their caregivers.

\section{Exploratory Analyses}

Patient Visual Avoidance and Caregiver Depression and Anxiety

Since rates of anxiety and depressive disorders are 2-4 times higher in dementia caregivers compared to age-matched noncaregiving controls [34-37], we conducted exploratory analyses to determine whether less patient visual avoidance was associated with greater caregiver depression and anxiety symptoms, as assessed by the depression and anxiety subscales of the SCL-90. Two separate multiple regression analyses were used with CDR Box Score as a covariate, patient visual avoidance as the predictor variable, and caregiver depression and anxiety scores as outcome variables (see Table 4 for caregiver means and standard deviations).

\section{Caregiver Depression}

To control for dementia severity, we entered the CDR Box Score in the first step. In the second step, patient visual avoidance accounted for additional variance $(F$ change $(1,79)=$ $4.02, p=0.048)$. In this second step, dementia severity did not predict caregiver depression $(t(79)=0.24, p=0.815)$, but less patient visual avoidance predicted greater caregiver depression $(\beta=-0.22, t(79)=-2.01, p=0.048)$.

\section{Caregiver Anxiety}

To control for dementia severity, we entered the CDR Box Score in the first step. In the second step, patient visual avoidance again accounted for additional variance $F$ change (1, 
$79)=4.37, p=0.040)$. In this second step, dementia severity did not predict caregiver anxiety $(\beta=0.05, t(79)=-0.04, p=0.966)$, but less patient visual avoidance significantly predicted greater caregiver anxiety $(\beta=-0.23, t(79)=-2.09, p=0.040)$.

Individual Visual Avoidance Codes as Predictors of Caregiver Psychological Distress

An exploratory multiple regression was also conducted to examine the relationship between individual visual avoidance codes and caregiver psychological distress. To control for dementia severity, we entered the CDR Box Score in the first step. Patient individual visual avoidance codes (i.e., head turn, head down, head up, head shake, gaze aversion, blinks, eyes closed) were entered in the second step and accounted for additional variance ( $F$ change ( 7 , $76)=2.27, p=0.037)$. In this second step, dementia severity did not predict caregiver psychological distress $(\beta=-0.00, t(76)=-0.03, p=0.979)$, but fewer patient headshakes (i.e., turning the head from side to side in a successive manner) predicted greater caregiver psychological distress $(t(76)=-2.09, p=0.040)$. In addition, less eye closures marginally predicted greater caregiver psychological distress $(\beta=-0.23, t(76)=-1.99, p=0.050)$.

\section{Discussion}

Using a laboratory-based assessment of visual avoidance in response to a disgust-eliciting film, we found that low levels of visual avoidance in dementia patients were associated with high levels of psychological distress in their familial caregivers. Meditational analyses revealed that this relationship was accounted for more by lower levels of emotional functioning (measured by caregiver report on the CASEF) than by lower levels of cognitive functioning (measured by caregiver report on the CDR cognitive subscales) or higher levels of neuropsychiatric symptoms (measured by clinician report on the NPI total score). This relationship was stronger in patients with bvFTD, a form of dementia associated primarily with declines in social and emotional functioning, than in patients with $\mathrm{AD}$, which is associated primarily with declines in cognitive functioning [38].

Exploratory analyses revealed that lower levels of visual avoidance among patients predicted higher levels of caregiver depression and anxiety. Lastly, considering all individual visual avoidance codes together revealed that only less patient headshakes was significantly associated with greater caregiver psychological distress, above and beyond patient disease severity.

\section{Implications for Understanding and Preventing Caregiver Psychological Distress}

With the aging population, rising prevalence of dementia, and increasing number of individuals providing care for dementia patients, it is critically important to understand the factors that contribute to vulnerability and resiliency to psychological distress in caregivers. This knowledge has implications both for understanding the etiology of mental illness in caregivers and for identifying potential targets for preventative and therapeutic interventions. Among these factors, patients' deficits in emotional regulation may be particularly important, given the importance emotion regulation has for adaptive interpersonal interactions [39] and the importance that social relationships have for mental health [40-43]. The present study focused on visual avoidance, a common form of emotion regulation that limits visual sensory input when we are confronted with a powerful emotional stimulus. We found that low levels of visual avoidance in patients when viewing a disgusting film were associated with greater psychological distress in their caregivers.

Although declines in patients' visual avoidance behaviors by themselves may be quite stressful for caregivers (e.g., it is unsettling when someone fails to follow social conventions 
regarding attention and inattention), our mediation analyses suggest that deficits in visual avoidance are associated with deficits in broader areas of patients' emotional functioning which, in turn, are associated with greater psychological distress in caregivers. These broader deficits could include declines in other emotional behaviors that are important for caregiver well-being such as reductions in patients' ability to empathize (e.g., inability to recognize emotions in others; Brown et al., under review), react appropriately to other kinds of emotional stimuli (e.g., diminished self-conscious emotion) [44], and regulate emotion in other ways (e.g., by suppression) [45]. Although the current study did not find that patient NPI total scores mediated the effect of patient visual avoidance on caregiver psychological distress, the negative impact of patient neuropsychiatric symptoms on caregiver well-being is well documented [46-50]. Thus, future studies will also benefit from examining how patient emotional functioning relates to broader neuropsychiatric symptomatology known to negatively influence caregivers, such as behavioral disinhibition (including sexual disinhibition), apathy, and aggression.

In considering the burden experienced by caregivers of patients with bvFTD and $\mathrm{AD}$, the behavioral changes in bvFTD can be particularly difficult for caregivers [51-55]. We expect that this is why the relationships among deficits in patients' visual avoidance, low levels of patients' emotional functioning, and high levels of caregiver psychological distress were stronger when patients had bvFTD than AD. Patients with bvFTD are more likely to show declines in broad areas of emotional functioning than patients with AD (at least in the early stage of the disease) $[56,57]$. Thus, declines in visual avoidance in patients with bvFTD are less likely to be offset by preservation of other areas of emotional functioning.

\section{Strengths and Limitations}

This study found that deficits in patients' visual avoidance behaviors when viewing a disgusting film were associated with greater psychological distress in caregivers. Strengths of the study included: (a) using an objective measure of visual avoidance; an important and often understudied form of emotion regulation; (b) studying patients with multiple kinds of dementia (bvFTD and AD); (c) having a relatively large sample size for this kind of patient research; and (d) considering both emotional, cognitive, and neuropsychiatric mediators. Limitations included: (a) only measuring avoidance behaviors to a single film stimulus and a single emotion (additional film stimuli would allow us to examine the generalizability of our findings), and (b) measuring all variables close in time (a longitudinal design would have been preferable both for evaluating possible directions of influence and for providing a stronger test of mediators). In terms of bidirectional influence, declines in patients' visual avoidance and associated declines in their overall emotional functioning are plausible contributors to caregivers' psychological distress. Conversely, declining psychological health in caregivers could contribute to declining visual avoidance in patients (e.g., via distracted caregivers providing less corrective feedback to patients).

\section{Conclusion}

The present study found that lower levels of patients' visual avoidance in response to a disgusting film were related to higher levels of caregiver psychological distress. With the rising rates of dementia and other neurodegenerative diseases, there will be increasing numbers of familial caregivers exposed to the challenges of caregiving. Thus, it is critically important to identify factors in patients, caregivers, and patient-caregiver relationships that influence vulnerability and resiliency of caregivers to mental and physical illness. 
Otero and Levenson: Lower Visual Avoidance in Dementia Patients Is Associated with Greater Psychological Distress in Caregivers

These factors can be used to identify individuals who are at heightened risk for negative outcomes associated with caregiving and as targets for preventative and therapeutic interventions.

\section{Acknowledgment}

This study was supported by the NIH under Grant R37AG017766. Preparation of the manuscript was supported by the National Institute of Aging Grant P01AG019724 and AG41762 to R.W.L. and a National Science Foundation Grant DGE 1106400 to M.C.O.

\section{Disclosure Statement}

The authors report no disclosures or conflicts of interest.

\section{References}

1 World Health Organization: Dementia a public health priority. 2012. http://site.ebrary.com/lib/ucmerced/ Doc?id=10718026.

2 Braak H, Braak E: Frequency of stages of Alzheimer-related lesions in different age categories. Neurobiol Aging 1997;18:351-357.

3 Greicius MD, Srivastava G, Reiss AL, Menon V: Default-mode network activity distinguishes Alzheimer's disease from healthy aging: evidence from functional MRI. Proc Natl Acad Sci USA 2004;101:4637-4642.

4 McKhann G, Drachman D, Folstein M, Katzman R, Price D, Stadlan EM: Clinical diagnosis of Alzheimer's disease: Report of the NINCDS-ADRDA Work Group under the auspices of Department of Health and Human Services Task Force on Alzheimer's Disease. Neurology 1984;34:939-944.

5 Knopman DS, Roberts RO: Estimating the number of persons with frontotemporal lobar degeneration in the US population. J Mol Neurosci 2011;45:330-335.

6 Onyike CU, Diehl-Schmid J: The epidemiology of frontotemporal dementia. Int Rev Psychiatry 2013;25:130137.

7 Ratnavalli E, Brayne C, Dawson K, Hodges JR: The prevalence of frontotemporal dementia. Neurology 2002; 58:1615-1621.

8 Neary D, Snowden JS, Gustafson L, Passant U, Stuss D, Black S, et al: Frontotemporal lobar degeneration: a consensus on clinical diagnostic criteria. Neurology 1998;51:1546-1554.

9 Piguet 0, Hornberger M, Mioshi E, Hodges JR: Behavioural-variant frontotemporal dementia: diagnosis, clinical staging, and management. Lancet Neurol 2011;10:162-172.

10 Pinquart M, Sörensen S: Differences between caregivers and noncaregivers in psychological health and physical health: a meta-analysis. Psychol Aging 2003;18:250-267.

11 Pinquart M, Sörensen S: Correlates of physical health of informal caregivers: a meta-analysis. J Gerontol B Psychol Sci Soc Sci 2007;62:P126.

12 Schulz R, Beach SR: Caregiving as a risk factor for mortality. JAMA 1999;282:2215.

13 Mioshi E, Bristow M, Cook R, Hodges JR: Factors underlying caregiver stress in frontotemporal dementia and Alzheimer's disease. Dement Geriatr Cogn Disord 2009;27:76-81.

14 Arango Lasprilla JC, Moreno A, Rogers H, Francis K: The Effect of dementia patient's physical, cognitive, and emotional/behavioral problems on caregiver well-being: findings from a Spanish-Speaking sample from Colombia, South America. Am J Alzheimers Dis Other Demen 2009;24:384-395.

15 Rosdinom R, Zarina MZN, Zanariah MS, Marhani M, Suzaily W: Behavioural and psychological symptoms of dementia, cognitive impairment and caregiver burden in patients with dementia. Prev Med 2013;57:S67S69.

16 Ryan KA, Weldon A, Persad C, Heidebrink JL, Barbas N, Giordani B: Neuropsychiatric symptoms and executive functioning in patients with mild cognitive impairment: relationship to caregiver burden. Dement Geriatr Cogn Disord 2012;34:206-215.

17 Gross JJ, Thompson RA: Emotion regulation: Conceptual foundations. Handbook of Emotion Regulation. New York, Guildford Press, 2007, pp 3-24.

18 Ochsner K, Gross J: The cognitive control of emotion. Trends Cogn Sci 2005;9:242-249.

19 Diehlschmid J, Pohl C, Ruprecht C, Wagenpfeil S, Foerstl H, Kurz A: The Ekman 60 Faces Test as a diagnostic instrument in frontotemporal dementia. Arch Clin Neuropsychol 2007;22:459-464. 
Otero and Levenson: Lower Visual Avoidance in Dementia Patients Is Associated with Greater Psychological Distress in Caregivers

20 Eckart JA, Sturm VE, Miller BL, Levenson RW: Diminished disgust reactivity in behavioral variant frontotemporal dementia. Neuropsychologia 2012;50:786-790.

21 Neary D, Snowden J, Mann D: Frontotemporal dementia. Lancet Neurol 2005;4:771-780.

22 Levenson RW, Ascher E, Goodkind M, McCarthy M, Sturm VE, Werner K: Laboratory testing of emotion and frontal cortex. Handb Clin Neurol, 2008;88:489-498.

23 Shiota MN, Levenson RWL: Effects of aging on experimentally instructed detached reappraisal, positive reappraisal, and emotional behavior suppression. Psychol Aging 2009;24:890.

24 Rothbart MK, Ziaie H, O’Boyle CG: Self-regulation and emotion in infancy. New Dir Child Dev 1992;55:7-23.

25 Smilek D, Carriere JSA, Cheyne JA: Out of mind, out of sight. Psychol Sci 2010;21:786-789.

26 Mason EC, Richardson R: Looking beyond fear: the extinction of other emotions implicated in anxiety disorders. J Anxiety Disord 2010;24:63-70.

27 Derogatis LR, Unger R: Symptom Checklist-90-Revised; in The Corsini Encyclopedia of Psychology. John Wiley \& Sons, Inc., 2010, http://dx.doi.org/10.1002/9780470479216.corpsy0970.

28 Derogatis LR, Savitz KL: The SCL-90-R and Brief Symptom Inventory (BSI) in primary care. Handbook of Psychological Assessment in Primary Care Settings, Mahwah, Lawrence Erlbaum Assoc Publ Ed, 2000, pp 297-334.

29 Morris JC: Clinical dementia rating: a reliable and valid diagnostic and staging measure for dementia of the Alzheimer type. Int Psychogeriatr 1997;9(suppl 1):173-176.

30 Ascher EA: From Lab to Life: Concordance between Laboratory and Caregiver Assessment of Emotion in Dementia; unpublished doctoral dissertation, University of California, Berkeley, 2012.

31 Cummings JL: The Neuropsychiatric Inventory Assessing psychopathology in dementia patients. Neurology 1997;48(5 Suppl 6):10S-16S

32 Ware JE Jr, Sherbourne CD: The MOS 36-item short-form health survey (SF-36). I. Conceptual framework and item selection. Med Care 1992;30:473-483.

33 Nunnaly J: Psychometric theory. New York, McGraw-Hill, 1978.

34 Joling KJ, van Marwijk HWJ, Veldhuijzen AE, van der Horst HE, Scheltens P, Smit F, van Hout HPJ: The two-year incidence of depression and anxiety disorders in spousal caregivers of persons with dementia: who is at the greatest risk? Am J Geriatr Psychiatry 2015;23:293-303.

35 Zarit S, Orr NK, Zarit JM: The hidden victims of Alzheimer's disease: families under stress. New York, New York University Press, 1985.

36 Schulz R, Visintainer P, Williamson GM: Psychiatric and physical morbidity effects of caregiving. J Gerontol 1990;45:P181-P191.

37 Brodaty H, Donkin M: Family caregivers of people with dementia. Dialogues Clin Neurosci 2009;11:217-228.

38 Levenson RW, Miller BL: Loss of cells - loss of self frontotemporal lobar degeneration and human emotion. Curr Dir in Psychol Sci 2007;16:289-294.

39 Gross JJ, John OP: Individual differences in two emotion regulation processes: implications for affect, relationships, and well-being. J Pers Soc Psychol 2003;85:348.

40 Schulz R, O’Brien AT, Bookwala J, Fleissner K: Psychiatric and physical morbidity effects of dementia caregiving: prevalence, correlates, and causes. Gerontologist 1995;35:771-791.

41 Schulz R, Visintainer P, Williamson GM: Psychiatric and physical morbidity effects of caregiving. J Gerontol 1990;45:P181-P191.

42 Cacioppo JT, Hughes ME, Waite LJ, Hawkley LC, Thisted RA: Loneliness as a specific risk factor for depressive symptoms: cross-sectional and longitudinal analyses. Psychol Aging 2006;21:140-151.

43 Kawachi I, Berkman LF: Social ties and mental health. J Urban Health 2001;78:458-467.

44 Sturm VE, Rosen HJ, Allison S, Miller BL, Levenson RWL: Self-conscious emotion deficits in frontotemporal lobar degeneration. Brain 2006;129:2508-2516.

45 Goodkind MS, Gyurak A, McCarthy M, Miller BL, Levenson RW: Emotion regulation deficits in frontotemporal lobar degeneration and Alzheimer's disease. Psychol Aging 2010;25:30.

46 Lima-Silva TB, Bahia VS, Carvalho VA, Guimares HC, Caramelli P, Balthazar ML, Yassuda MS: Neuropsychiatric symptoms, caregiver burden and distress in behavioral-variant frontotemporal dementia and Alzheimer's disease. Dement Geriatr Cogn Disord 2015;40:268-275.

47 Bjoerke-Bertheussen J, Ehrt U, Rongve A, Ballard C, Aarsland D: Neuropsychiatric symptoms in mild dementia with Lewy bodies and Alzheimer's disease. Dement Geriatr Cogn Disord 2012;34:1-6.

48 Rocca P, Leotta D, Liffredo C, Mingrone C, Sigaudo M, Capellero B, Bogetto F: Neuropsychiatric symptoms underlying caregiver stress and insight in Alzheimer's disease. Dement Geriatr Cogn Disord 2010;30:57-63.

49 Massimo L, Powers C, Moore P, Vesely L, Avants B, Gee J, Grossman M: Neuroanatomy of apathy and disinhibition in frontotemporal lobar degeneration. Dement Geriatr Cogn Disord 2009;27:96-104.

50 Matsumoto N, Ikeda M, Fukuhara R, Shinagawa S, Ishikawa T, Mori T, Tanabe H: Caregiver burden associated with behavioral and psychological symptoms of dementia in elderly people in the local community. Dement Geriatr Cogn Disord 2007;23:219-224.

51 Mourik JC, Rosso SM, Niermeijer MF, Duivenvoorden HJ, van Swieten JC, Tibben A: Frontotemporal dementia: behavioral symptoms and caregiver distress. Dement Geriatr Cogn Disord 2004;18:299-306.

52 Ascher EA, Sturm VE, Seider BH, Holley SR, Miller BL, Levenson RW: Relationship satisfaction and emotional language in frontotemporal dementia and Alzheimer's disease patients and spousal caregivers. Alzheimer Dis Assoc Disord 2010;24:49. 
Otero and Levenson: Lower Visual Avoidance in Dementia Patients Is Associated with Greater Psychological Distress in Caregivers

53 de Vugt ME, Riedijk SR, Aalten P, Tibben A, van Swieten JC, Verhey FRJ: Impact of behavioural problems on spousal caregivers: a comparison between Alzheimer's disease and frontotemporal dementia. Dement Geriatr Cogn Disord 2006;22:35-41.

54 Riedijk SR, De Vugt ME, Duivenvoorden HJ, Niermeijer MF, van Swieten JC, Verhey FRJ, Tibben A: Caregiver burden, health-related quality of life and coping in dementia caregivers: a comparison of frontotemporal dementia and Alzheimer's disease. Dement Geriatr Cogn Disord 2006;22:405-412.

55 Wong C, Merrilees J, Ketelle R, Barton C, Wallhagen M, Miller B: The experience of caregiving: differences between behavioral variant of frontotemporal dementia and Alzheimer disease. Am J Geriatr Psychiatry 2012; 20:724-728.

56 Freedman M, Binns MA, Black SE, Murphy C, Stuss DT: Theory of mind and recognition of facial emotion in dementia: challenge to current concepts. Alzheimer Dis Assoc Disord 2013;27:56-61.

57 Lee GJ, Lu PH, Mather MJ, Shapira J, Jimenez E, Leow AD, Mendez MF: Neuroanatomical correlates of emotional blunting in behavioral variant frontotemporal dementia and early-onset Alzheimer's disease. J Alzheimers Dis 2014;41:793-800. 